\title{
Patterns with Symmetries of the Wallpaper Group on the Hyperbolic Space
}

\author{
Peichang Ouyang ${ }^{\mathrm{a}}$, Zhanglin $\mathrm{Li}^{\mathrm{b}^{*}}$ and Tao $\mathrm{Yu}^{\mathrm{a}}$ \\ ${ }^{a}$ School of Mathematics and Physics, Jinggangshan University; ${ }^{b}$ Computer Faculty, China University of Geosciences, \\ China
}

\begin{abstract}
Equivariant function with respect to symmetries of the wallpaper group is constructed by trigonometric functions. A proper transformation is established between Euclidean plane and hyperbolic spaces. With the resulting function and transformation, wallpaper patterns on the Poincaré and Klein models are generated by means of dynamic systems. This method can be utilized to produce infinity of beautiful pattern automatically.
\end{abstract}

Keywords: Hyperbolic geometry, klein model, poincaré model, wallpaper groups,

\section{INTRODUCTION}

Patterns that have symmetries of the wallpaper groups (or crystallographic group) can be found widely in the ancient decorative arts. However, the serious study of such groups is of comparatively recent. In 1924, Hungarian mathematician first listed the 17 wallpaper groups [1,2]. It is surprising that 230 crystallographic groups in three dimensional Euclidean spaces were discovered before planar wallpaper groups. With the development of computer techniques, there are many methods dedicated to the automatic generation of symmetric patterns. For example, in $[3,4]$, colorful images with symmetries of wallpaper groups were considered from the view point of dynamic systems. Many nice chaotic wallpaper attractors were produced by iterating equivariant functions [5-10].

Hyperbolic geometry differs from the familiar Euclidean geometry merely in how it handles the parallel axiom: given a line and a point outside it, more than one parallel line through the point exists [11]. Compared with Euclidean patterns, the visualization of hyperbolic patterns are rather unusual but exotic. In 1986, Dunham first utilized computers to create hyperbolic patterns automatically [12]. Inspired by his idea, various approaches have been investigated to create hyperbolic patterns [13-18].

It is well known that Euclidean and hyperbolic patterns are incompatible since they have essential differences [1921]. However, by establishing proper transformations, we present a way to create wallpaper patterns on the hyperbolic space. To our knowledge, this approach has never been reported in any literature so far.

The reminder of this paper is organized as follows. In Section 2, we present a method to yield patterns with symmetries of the wallpaper groups. In Section 3, to create Euclidean patterns on hyperbolic space, we establish a proper transformation between Euclidean and hyperbolic spaces. In Section 4, we describe how to yield wallpaper patterns on hyperbolic models and show some detailed implements.

\section{FUNCTIONS THAT ARE EQUIVARIANT WITH RESPECT TO WALLPAPER GROUPS}

In this section, we present a simple method to construct functions that are equivariant with respect to wallpaper groups.

A function is said to be equivariant with respect to the symmetries of $\mathrm{G}$ if for all,, where .

Since wallpaper groups are characterized by translation in two independent directions, which give rise to a lattice. We need to consider a lattice in the plane along with a dual lattice. This means that, if and, then should be an integer. So if and, then is an integer multiple of and.

Supposeare real constants, then is equivariant with respect to the translations. Here the notation means to design reduction of points via the lattice to a fundamental field. With these analyses, we obtain the following proposition immediately.

Proposition 1. Let be a finite subset of,,, A, are real constants, then is equivariant with respect to the translational symmetries of .

By periodicity, it is easy to check that Proposition 1 holds if we interchange sine or cosine arbitrarily.

For wallpaper groups, we need two kind lattices. One is square fundamental field with base and, which is self-dual. We use. The other is hexagonal lattice, the base can be chosen as. So we obtain a different dual lattice.

Proposition 2 [6]. Let be an arbitrary function, be a finite group realized by matrices acting on. Assume is a function defined as

Then, is equivariant with respect to. 
Theorem 3. Let be a function defined as (1), be a group that maps the lattice back onto itself. Suppose is a function defined in Proposition 2. Then is equivariant with respect to translation symmetries of and symmetries of.

Let and be, respectively, cyclic and dihedral groups. Denote and. Then is the generator of and is the realization of. We will consider being equal to that set of matrices. Since is a reflection through the origin, set is a presentation of group.

\section{TRANSFORMATIONS BETWEEN EUCLIDEAN PLANE AND HYPERBOLIC SPACE}

Hyperbolic geometry was established by Lobachevsky, Bolyai and Gauss at the beginning of $19^{\text {th }}$ century [19-21]. There are many different models of hyperbolic geometry in Euclidean space. In this section, we establish transformations between Euclidean plane and hyperbolic models. We only consider the three most familiar hyperbolic models: Poincaré, Klein and upper half-plane models [21].

The definition domain of Poincaré and Klein models is the point in the interior of the Euclidean unit disk, i.e.,. Notice that the hyperbolic lines in Poincaré model are circular arcs cut the boundary of the disk at right angle; while hyperbolic lines in Klein model are interpreted as chords of the unit disk (including diameters).

The definition domain of upper half-plane model is the point of the upper half plane, i.e.,. In this model, hyperbolic lines are semicircles orthogonal to the -axis, including vertical lines.

The formula that transforms Klein model to upper halfplane model is

where.

The formula that transforms Poincaré model to upper half-plane model is

where [22].

It is well known that Euclidean and hyperbolic patterns are incompatible since they have different metric. In some sense, they are the completely distinct worlds. However, if we treat upper half-plane model as Euclidean space, then (3) and (4) establish transformations linking the two spaces. This provides a way to create Euclidean patterns on the hyperbolic model.

The practical coordinates used in experiment were magnified by. For example, the coordinates used in (3) are. Let be the mesh formed by mutually perpendicular linesand. By using the inverse transformation of formula (4), i.e., where, we obtain a corresponding mesh on the Poincaré model, as shown in Fig. (1). Similarly, we can also generate the mesh on the Klein model.

\section{PATTERNS WITH SYMMETRIES OF THE WALL- PAPER GROUPS ON THE HYPERBOLIC MODELS}

In this section, we describe how to create wallpaper patterns on the Poincaré and Klein models.

We take Klein model as an example showing how to create aesthetic patterns on hyperbolic space. We summarize it as follows:

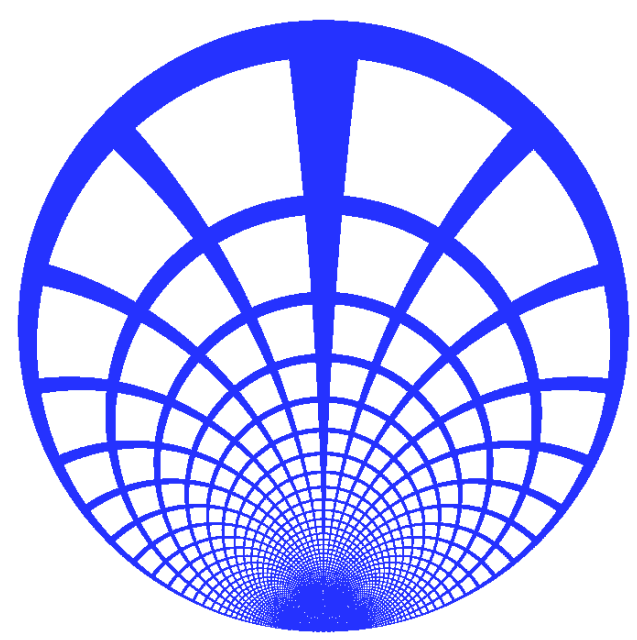

Fig. (1). The mesh on the Poincaré model obtained by transforming rectangular mesh of upper half-plane model.

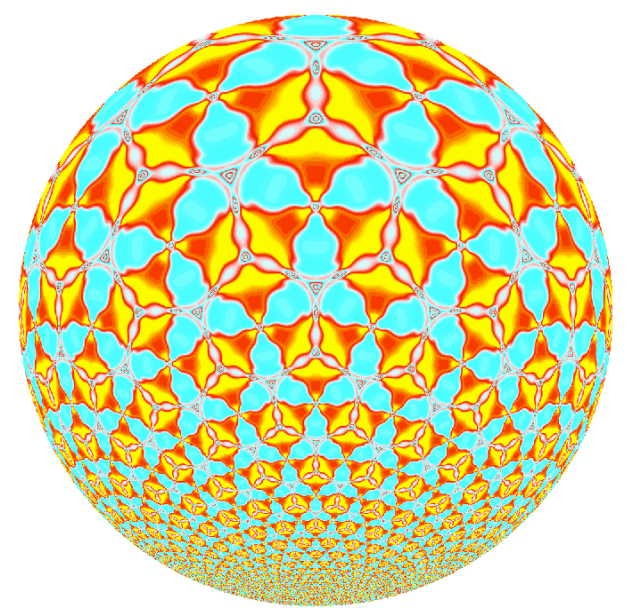

Fig. (2). An aesthetic pattern of kind.

Step 1. Let be a point of the unit disc on the Klein model. By formula (4), is transformed to the point on the upper halfplane.

Step 2. is used as initial iteration point of the equivariant function of theorem 3 .

Step 3. Use a certain color scheme to determine the color of. In this manner, we finally get a colored pattern on hyperbolic model which possesses the symmetries of wallpaper group.

The color scheme used in Step 3 was developed by Lu et al. They employed it to render wallpaper patterns [4]. For more details of the scheme, please refer to [4] and references therein.

For convenience, the caption donation "A_B" labeled in each figure means $A$ is the name of wallpaper group and $B$ is the kind of hyperbolic models. For example, pattern of the kind means it is a pattern in the Poincaré model with symmetry.

In Figs. (2-8) illustrate some wallpaper patterns on the hyperbolic modes. As a matter of fact, the patterns shown in Poincaré and Klein models have nested structures similar to the self-similarity of fractals. This could be firmed by magnifying figures. In Fig. (3), we show the magnified semicircle part of Fig. (2). 


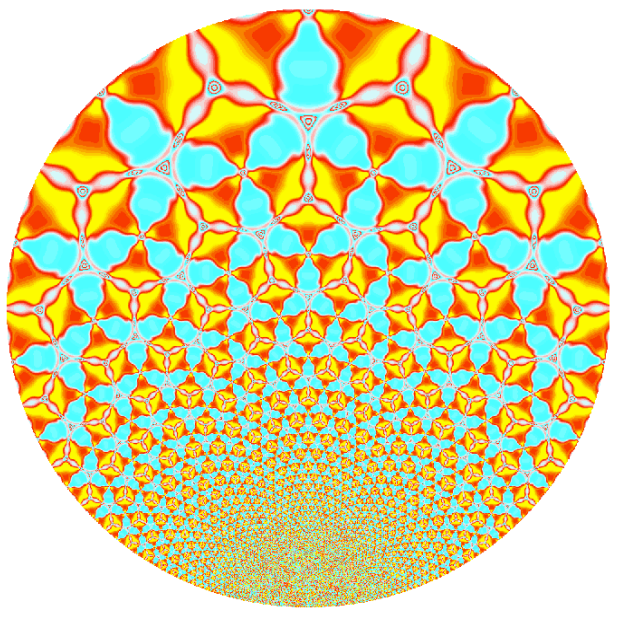

Fig. (3). An aesthetic pattern of kind.

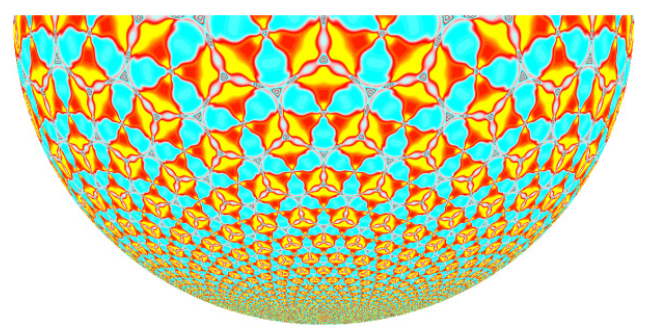

Fig. (4). The magnified semicircle of Fig. (2).

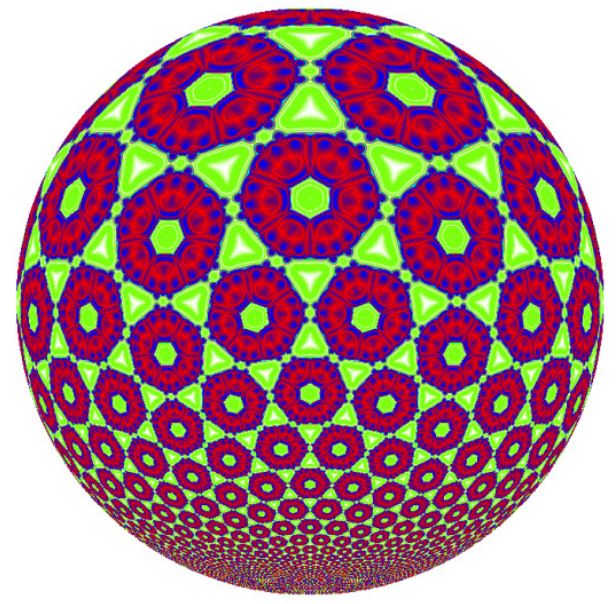

Fig.(5). An aesthetic pattern of kind.

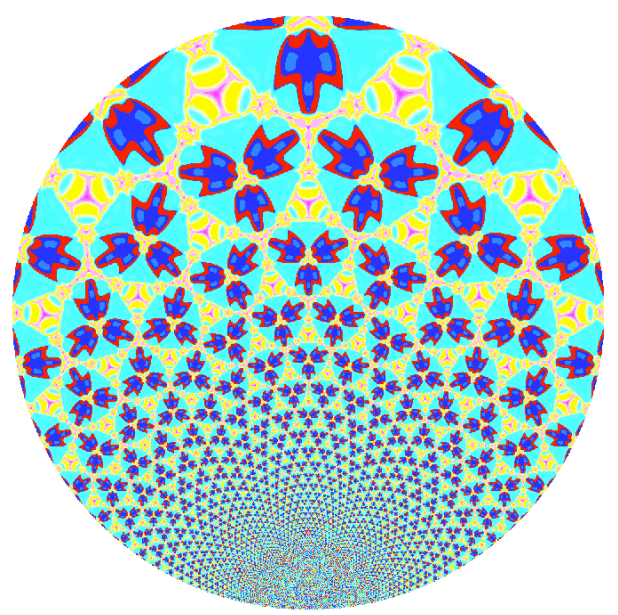

Fig. (6). An aesthetic pattern of kind.

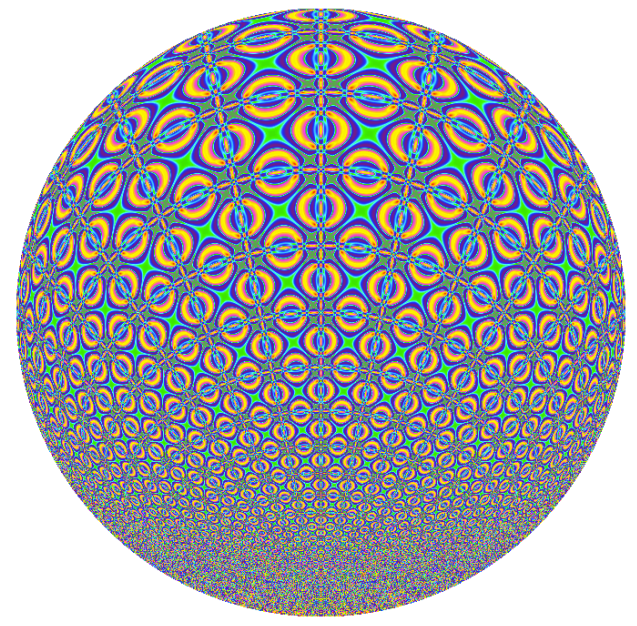

Fig. (7). An aesthetic pattern of kind.

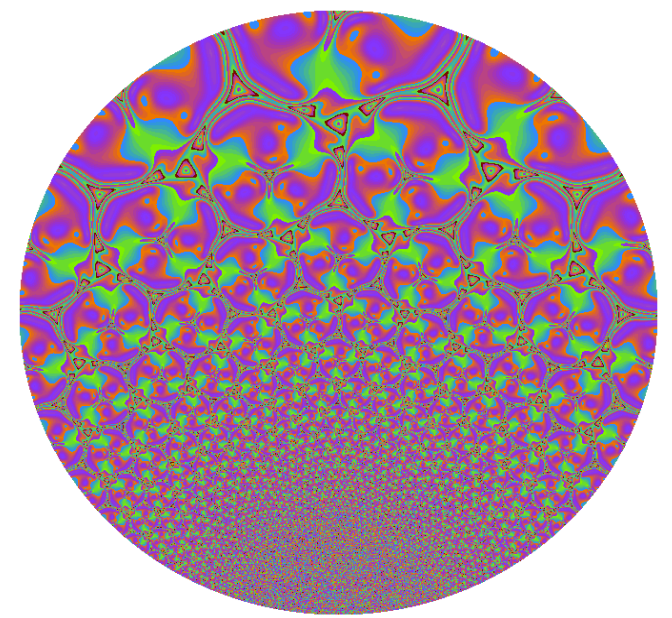

Fig. (8). An aesthetic pattern of kind .

\section{CONCLUSITON}

In this paper, we present a new form of equivariant functions with respect to wallpaper groups. We establish transformations which can transform Euclidean patterns into hyperbolic models perfectly. Though Euclidean and hyperbolic patterns are incompatible, our transformations can create aesthetic patterns on hyperbolic space, which realize the notion of infinity in a finite area. This method can create a great many of aesthetic patterns.

\section{CONFLICT OF INTEREST}

The authors confirm that this article content has no conflict of interest.

\section{ACKNOWLEDGEMENTS}

This work was supported by the Natural Science Foundation of China (No. 11461035), Natural Science Foundation of Jiangxi province of China (No. 20142BAB211012, No. 20132BAB211018).

\section{REFERENCE}

[1] B. Grünbaum, G.C. Shephard, Tilings and Patterns. W. H. Freeman and Company, New York, 1992. 
[2] M.A. Armstrong, Groups and symmetry. Springer-Verlag, New York, 1998.

[3] K.W. Chung, H.S.Y. Chan, "Symmetrical patterns from dynamics," COMPUT GRAPH FORUM, vol. 12, pp. 33-34, 1993.

[4] J. Lu, Z.X. Ye, Y.R. Zou, R.S. Ye, "Orbit trap rendering methods for generating artistic images with crystallographic symmetries," COMPUT GRAPH-UK, vol. 29, pp.787-794.

[5] M. Field, M. Golubitsky, Symmetry in Chaos. Oxford University Press, 1992.

[6] N. Carter, R. Eagles, S. Grimes, A. Hahn, C. Reiter, "Chaotic attractors with discrete planar symmetries," CHAOS SOLITON FRACT, vol. 12, pp. 2031-2054, 1998

[7] N. Carter, C. Reiter, "Frieze and wallpaper chaotic attractors with a polar spin," COMPUT GRAPH-UK, vol. 22, pp.765-779.

[8] J. Dumont, F. Heiss, K. Jones, C. Reiter, L. Vislocky, "Chaotic attractors and evolving planar symmetry," COMPUT GRAPH-UK, vol. 23, pp. 613-619, 1999.

[9] J. Dumont, C.A., Reiter, "Chaotic attractors near forbidden symmetry," CHAOS SOLITON FRACT, vol. 11, pp. 1287-1296,2000.

[10] N. Chen,F.C. Meng, "Critical points and dynamics systems with planar hexagonal symmetry," CHAOS SOLITON FRACT, vol. 32, pp. 1027-1037,2007.

[11] J.W. Anderson, Hyperbolic geometry. Springer-Verlag, New York, 2000.

[12] C.K. David, College Geometry. Holt, Rinehart and Winston, Inc 1969.

[13] D. Dunham, "Hyperbolic symmetry," COMPUT MATH APPL, vol. 12(B), pp. 139-153,1986.
[14] K.W. Chung, H.S.Y Chan, "Hyperbolic symmetries from dynamics," COMPUT MATH APPL, vol. 31, pp. 33-47,1996.

[15] K.W. Chung, B.N. Wang, "Tessellations with symmetries of the triangle groups from dynamics," INT J BIFURCAT CHAOS, vol. 13, pp. 3505-3518, 2003.

[16] K.W. Chung, H.S.Y Chan, B.N. Wang, "Tessellations with symmetries of the wallpaper groups and the modular group in the hyperbolic 3-space from dynamics," COMPUT GRAPH-UK, vol. 25, pp. 333-341, 2001

[17] K.W. Chung, H.S.Y Chan, B.N. Wang, "Efficient generation of hyperbolic symmetries from dynamics," CHAOS SOLITON FRACT, vol. 13, pp. 1175-1190, 2002.

[18] M. Adcock, K. Jones, C.A. Reiter, L. Vislocky, "Iterated function systems with symmetry in the hyperbolic plane," COMPUT GRAPH-UK, vol. 24, pp. 791-796, 2000

[19] N. Chen, D. Hao, M. Tang, "Automatic generation of symmetric IFS contracted in the hyperbolic plane," CHAOS SOLITON FRACT, vol. 41, pp. 829-842, 2009

[20] H.S.M. Coxeter, W.O.J. Moser, Generators and Relations for Discrete Groups. Springer-Verlag, New York, 1980.

[21] R.J. Trudeau, The non-Euclidean revolution. Birkhauser, Boston ,1987.

[22] M.J. Greenberg, Euclidean and non-Euclidean geometry. W. H. Freeman and company, 1993.

[23] B. Hausmann, B. Slopianka, H.P. Seidel, "Exploring plane hyperbolic geometry," Visualization and Mathematics, pp. 21-36, 1997.C. Reiter, "Attractors with dueling symmetry," COMPUT GRAPH-UK, vol. 21, pp. 263-271, 1997.

(C) Ouyang et al.; Licensee Bentham Open.

This is an open access article licensed under the terms of the Creative Commons Attribution Non-Commercial License (http://creativecommons.org/licenses/by-nc/3.0/) which permits unrestricted, non-commercial use, distribution and reproduction in any medium, provided the work is properly cited. 